\title{
Implementation of news module for news client based on ApiCloud
}

\author{
Xin $\mathrm{Fu}^{1, \mathrm{a},}, \mathrm{Yu}$ Liang ${ }^{2, \mathrm{~b}}$, Sanxing $\mathrm{Cao}^{2, \mathrm{c}}$, Hongbo $\mathrm{Gu}^{3, \mathrm{~d}}$ \\ ${ }^{1}$ Electronic and Communication Engineering, Communication Unversity of China, Beijing, China \\ ${ }^{2}$ New Media Institute, Communication Unversity of China, Beijing, China \\ ${ }^{3}$ Information center, State Forestry Administration of China, Beijing, China
}

\begin{abstract}
With the development of new media technology, news client has become the main battlefield of news browsing. Based on the ApiCloud hybrid development platform, this paper uses HTML, JavaScript and other technologies to develop the mobile client news module, and uses WAMP integrated development environment to build a news publishing system on the server side.
\end{abstract}

\section{INTRODUCTION}

The traditional native development of App needs to use a platform-specific language for development. iOS platform using Objective-C language, Android platform using Java language, to generate a specific client to adapt to the platform. Web applications uses the embedded terminal browser, front-end uses HTML, CSS, interactive response through the JavaScript to achieve mobile application

Mobile clients are mainly divided into three types: native, web and hybrid development. ApiCloud consists of two parts: cloud API and port API, which can help developers quickly realize the whole lifecycle management of mobile applications, including development, testing, publishing, management and operation. The overall architecture of the ApiCloud is shown in figure 1 .

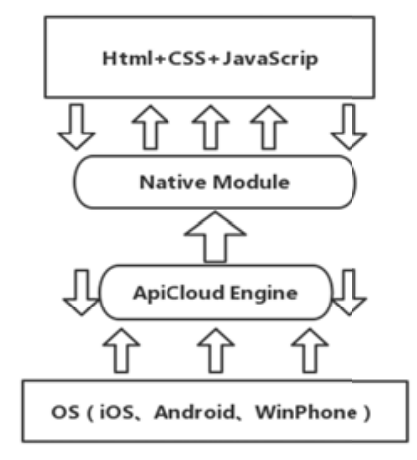

Fig. 1. Overall architecture of the ApiCloud

\subsection{Apache server}

development. The hybrid application development program adopted in this paper is the ApiCloud development platform, which implements the functions of news uploading, news channel setting and other functional modules.

\section{INTRODUCTION OF RELATED TECHNICAL}

\subsection{APICLOUD}

Apache HTTP Server (Apache) is a Web server software, its utilization rate ranked first in the world. In almost all the computer platform, Apache can run well. It is fast and reliable and has a powerful characteristics of crossing platform. It performs stably and it supports Web server applications which applied crossing platform, and it is widely used by many developers and websites.

\subsection{PHP}

PHP (Hypertext Preprocessor, hypertext preprocessor) is a universal open source scripting language, the file suffix called .php, which combines the C language, Perl and Java features. You can access the database using PHP. PHP is suitable for the field of Web development, it can carry out dynamic webpage faster, more efficient than other programming languages. Due to the Web system is to embed the program into HTML, and it almost matches all of the current database, such as MySQL, Oracle, Sysbase, SQLServer, dBase etc.

\subsection{MySQL database}

MySQL is an association database management system 
developed by Swedish MySQL AB. Instead of setting all the data together, but the data would be stored in different tables, so as to speed up the access speed, and clear the database structure design, improve the flexibility of database structure. MySQL has the characteristics of open source, small size and fast speed. It is widely used in all kinds of systems under the Internet environment. It can match Apache and PHP to form a development environment with excellent performance at the same time.

\subsection{Apache+PHP+MySQL}

In this paper, the $\mathrm{B} / \mathrm{S}$ architecture is used to implement the system. In the Browser, based on ApiCloud, the client interfaces display and user interaction functions. Server uses PHP to complete the implementation of functional components, in order to complete most of the function in logic. And the Server uses easily developed environment XAMPP as the operating environment. XAMPP (Apache+MySQL+PHP) is a full-featured integrated development environment in Web, it can run on the platform of Windows, OSX, and Linux, cross platform superior. News client is based on Windows APP background Server 2012 as the operating system, using Apache as Web server, MySQL database, development language is PHP, which can control the connection with database and ApiCloud client package.

\section{Demand analysis for news client}

\subsection{Demand of front-end interface}

In the front end of the news client, the home page is the most important and complex page. The news client designed in this paper contains the top and bottom navigation bars. The navigation bar contains many categories, such as headline, hot spot, Beijing and so on. The middle part of the home page is a thumbnail display of graphic news and video news. News is divided into graphic news and video news. Click on the news list, access to landing news page. At the bottom of the atlas are news sources, times, comments, and information.

At the same time, the user's personal center page is also an indispensable demand in the news client. The client's personal center page draws on the design of the NetEase news client. Including user login, user registration, news collection and other functions.

\subsection{News release system}

The client's news release system, news shown on the front end all need to edit the news materials uploaded to the database for client to read, display and use. The news includes pictures, news and video news. User reviews management is also the function of this system, which can delete the user's unqualified comments. These are the core components of server-side code.

In this news release system, the administrator logins in, inputs the administrator name and password to enter into the editing system, then they can edit text news, video news. And the system will store the operation information of administrator. The detailed development module will be described in the fourth chapter. The overall requirements architecture relationship of this news client is shown in figure 2 .

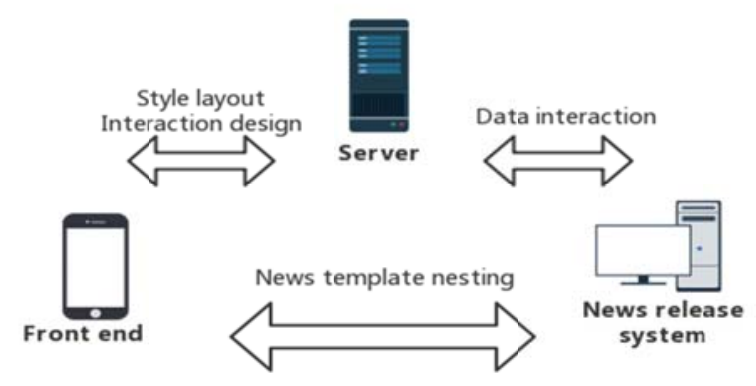

Fig. 2. Total demand framework

\section{4 the design and implementation of news client module}

The core of the news client is the news release system, and all the news content displayed at the front end is edited and uploaded through the background, and finally appeared at the front end interface. The background system of the news release system for editors includes uploading news, adding, deleting, modifying, user reviews management and other functions. The back-end server mainly handles various requests sent by the mobile client, and realizes the front presentation of the data.

The news client is divided into 4 modules, each module is responsible for different functions.

\subsection{Channel settings module}

The news client news is designed in different channels. The news channel setting system includes three parts: the setting of the channel, the modification of the channel name and the deletion of the channel.

The web page transmits the form through the post and passes the data to the addCate function in the doAminAction.php file, where the addCate function receives the data and writes the data into the database, form transfers code. Create article table storage uploaded news according to the demand, which sets field ID (int) as the primary key, the video thumbnails and memory address. Using ',' connected them into a string, and then stor tag (varchar) said that this news is hot or recommended, title (varchar) said the news' headlines, content (text) said the 
theme of the news' text, imgs (varchar) said the news' thumbnails and video address, type (varchar) said the news' category, editdate (varchar) said the news' editor of the date, channel (varchar) said that belongs to which channel, editor (varchar) said for authors and editors, imgsarticle (varchar) said the thumbnails and video upload. While imgs and imgsarticle all represent the thumbnail, but their contents are not the same, the former is filtered. The latter is extracted, APP reads directly when the client request, and imgsarticle is the address uploaded through local without filtering. The specific settings are shown in Table1.

Table 1. Setting of database

\begin{tabular}{|c|c|c|c|}
\hline $\begin{array}{c}\text { Attribute } \\
\text { name }\end{array}$ & Type & $\begin{array}{c}\text { Allow for } \\
\text { empty }\end{array}$ & Auto_INCREMENT \\
\hline id & int(8) & no & Yes \\
\hline tag & varchar(10) & no & Yes \\
\hline title & varchar(50) & no & Yes \\
\hline content & text & no & Yes \\
\hline imgs & text & no & Yes \\
\hline type & varchar(10) & no & Yes \\
\hline editdate & date & no & Yes \\
\hline channel & varchar(20) & no & Yes \\
\hline editor & varchar(10) & no & Yes \\
\hline imgsarticle & text & no & Yes \\
\hline
\end{tabular}

\subsection{News upload module}

The news upload module is the core of background editing, background administrator can upload news by setting the account after landing good news channel, including graphic news and video news. Different news can be related to different channels. Graphic news uploading includes thumbnails of pictures, news headlines, text editors, editors, authors, date settings, marking news for hot spots, and recommendations. Video news uploading includes video thumbnails, news headlines, text editors, editors, authors, date settings, marking news for hot spots, and recommendations.

The data uploaded by video news achieves the transmission by the form, and uploads the video to the specified folder and rename through the KindEditor. And choosing which channel the video is stored on, needs to retrieve the data stored in the database in channel, traverse it, and then store it in the select tag for selection. And accepting form data addProl function, accepting thumbnails and video data needs to extract the two from the KindEditor raw data, rearrange them, and then deposit them into the database, which is applied to regular expressions. By using regular expressions to extract the memory address of the uploaded thumbnails and video, and then deposit the string connected by ',' into the field of imgs. The client reading the field is only need to use the "split" function, it can separate thumbnail address and video address.

Graphic news uploads and video news uploads are about the same, but there are differences, thumbnails. Only need to upload one thumbnail news video can, and graphic information in the client has two display modes, one thumbnail mode and three thumbnail mode, which requires the need to determine the number of thumbnail stored in the database. More than three pieces is automatically selected three picture mode. The extraction of image address is through regular expressions, while extracted array automatically extracted data using the "," to connect. Single array directly transfer into a string and stored in the imgs field. The client first split the string in the imgs field by 'split' function when request comes, while the array number is greater than 2 , it comes to the three display mode, the number of 1 or 2 when using only the first array value as a thumbnail display address.

\subsection{User comments management module}

After registered users logging in the news client, you can use four functions including comments, modifying personal information, collection news, sharing news. For some users' inappropriate comments, the system administrator from the background landing system has the right to delete user' comments, but did not permit to modify the users' comments.

\subsection{Server response module}

The server is mainly used to store CSS style files and JavaScript interactive files designed at the front end, as well as news and databases. Under normal circumstances, using AJAX for cross domain requests is prohibited because of security problems, so you should make a change in the server file. Each PHP file needs to have header ('Access-Control-Allow-Origin: *) statement, the statement that allows for cross domain requests.

Each entity in the database is associated with the overall E-R diagram, as shown in Figure 3 below.

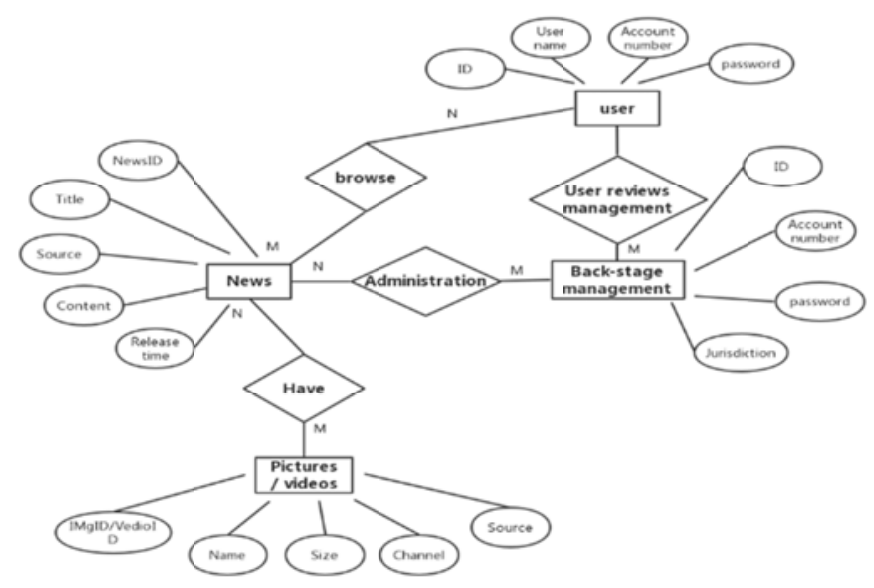


Fig. 3. Database entity association E-R diagram

\section{Conclusion}

The news client implemented in this paper is a new APP designed for iOS and Android smart phones and other intelligent mobile terminal user users. In the design aspect, using the flat design conforms to the handset user's operation custom. In terms of technology, the use of advanced HTML5+CSS3+JS technology sends mobile phone users to a light, smooth operation experience. In terms of function, compared to the PC terminal, the content structure is rearranged and designed to make it more concise and meticulous, more focused on content reading, more suitable for touch screen operation.

\section{Acknowledgements}

The work is sponsored by China Forestry Mobile Internet Development Strategy Research Project (2016 - R23) granted by State Forestry Administration Information Center and New Media Research Institute of Communication University of China.

\section{REFERENCE}

1. Wei Yabin. Design and implementation of mobile game news system based on HTML5[D]. Zhejiang University of Technology, 2015.

2. Guo Jundong. Design and implementation of recommender system based on wireless news client [D]. Shandong University, 2016.

3. $\mathrm{Hu}$ Yonghua, Dai Lin. Design and implementation of mobile campus news client [J]. information communication, 2015 (1): 110-111.

4. Li Yuan, Nie Yubao. IPhone platform network news client time module based on [J]. Journal of Shaoguan University, 2012, 33 (6): 18-22.

5. Li Ting. Design and implementation of science and technology news recommendation system based on iOS platform [D]. Dalian University of Technology, 2015.

6. Ding Nan. Research on mobile products based on HTML5 and CSS3's NetEase WAP touch screen version [D]. Huazhong University of Science and Technology, 2012.

7. Lane D. Web Database Applications with Php and Mysql[M]// Web database applications with php and mysql, second edition. O'Reilly Media, Inc. 2004.

8. Ding S Y, Cheng Z Q. Android Client Development based on Moodle Platform[J]. International Journal of Advanced Science \& Technology, 2016,87 